\title{
Manuseio de Grave Diminuição de Hemoglobina em Paciente Jovem, Testemunha de Jeová, Submetido à Proctocolectomia Total. Relato de Caso *
}

\section{Extreme Intraoperative Hemodilution in Jehovah's Witness Patient Submitted Total Proctocolectomy. Case Report}

Luiz Eduardo Imbelloni, TSA ${ }^{1}$; Lúcia Beato, TSA ${ }^{1}$; Arídio Ornellas ${ }^{2}$; Carlos Roberto Junqueira Borges ${ }^{3}$

\begin{abstract}
RESUMO
Imbelloni LE, Beato L, Ornellas A, Borges CRJ - Manuseio de Grave Diminuição de Hemoglobina em Paciente Jovem, Testemunha de Jeová, Submetido à Proctocolectomia Total. Relato de Caso
\end{abstract}

JUSTIFICATIVA E OBJETIVOS: Os riscos de transfusão homóloga de sangue são bem conhecidos e alguns pacientes recusam esta transfusão por motivos religiosos. O objetivo foi relatar um caso de proctocolectomia total em Testemunha de Jeová onde o nível de hemoglobina foi de $4 \mathrm{~g} / \mathrm{dL}$.

RELATO DO CASO: Paciente do sexo masculino, 17 anos, história de polipose intestinal familiar. Iniciada aos oito anos, caracterizada por sangramento. Aos 13 anos colectomia total. Aos 17 anos proctocolectomia total. Preparado com eritropoietina, ácido fólico, infusão de ferro e vitamina $B_{12}$. Hemograma revelou: hemácias $4.200 .000 / \mathrm{mm}^{3}$, hemoglobina $10,5 \mathrm{~g} / \mathrm{dL}$ e hematócrito de $37 \%$. Plaquetas $273.000 / \mathrm{mm}^{3}$, tempo de protrombina normal. Monitorização com PANI, oximetria de pulso, capnografia e ECG continuamente. Anestesia com propofol, sufentanil, pancurônio e enflurano em circuito fechado. Infusão de $7.000 \mathrm{~mL}$ de solução de Ringer com lactato e $150 \mathrm{~mL}$ de albumina humana a 20\%. Diurese de $2.900 \mathrm{~mL}$. Duração de 10 horas e 30 minutos. Na UTI Ht de $20 \%$, hemácias $2.300 .000 / \mathrm{mm}^{3}, \mathrm{Hb}$ de $4,2 \mathrm{~g} / \mathrm{dL}$ e mantido com propofol e atracúrio. Exame no dia seguinte revelou: Ht de horas após o término da cirurgia. Segundo dia encaminhado para o quarto. Quarto dia iniciada alimentação por via oral. Alta hospitalar no décimo dia de PO. No $30^{\circ} \mathrm{PO} \mathrm{Ht}$ de $35 \%$, hemácias de 4.000.000/ $\mathrm{mm}^{3}$ e $\mathrm{Hb}$ de $9,5 \mathrm{~g} / \mathrm{dL}$. Seis meses após, fechamento da ileostomia. Submetido a 12 cirurgias sem transfusão sangüínea.

CONCLUSÕES: Um planejamento de toda a equipe (clínico, cirurgião, anestesiologista e médicos de terapia intensiva) permite realizar procedimentos cirúrgicos associados com importantes perdas sangüíneas, sem administração de sangue.

\footnotetext{
${ }^{*}$ Recebido da (Received from) Clínica São Bernardo, Rio de Janeiro, RJ

1. Anestesiologista da Clínica São Bernardo

2. Chefe das Clínicas Cirúrgicas da Clínica São Bernardo

3. Chefe da Clínica Médica da Clínica São Bernardo
}

Apresentado (Submitted) em 27 de janeiro de 2005

Aceito (Accepted) para publicação em 09 de junho de 2005

Endereço para correspondência (Correspondence to)

Dr. Luiz Eduardo Imbelloni

Av. Epitácio Pessoa, 2356/203 Lagoa

22411-072 Rio de Janeiro, RJ

E-mail:dr.imbelloni@terra.com.br

(C) Sociedade Brasileira de Anestesiologia, 2005 $18 \%$, hemácias de 2.050.000/ $\mathrm{mm}^{3}, \mathrm{Hb}$ de $4 \mathrm{~g} / \mathrm{dL}$. Extubado 18

Unitermos: COMPLICAÇÕES: anemia; DOENÇAS: polipose

intestinal familiar; TRANSFUSÃO: testemunha de Jeová

\section{SUMMARY}

Imbelloni LE, Beato L, Ornellas A, Borges CRJ - Extreme Intraoperative Hemodilution in Jehovah's Witness Patient Submitted Total Proctocolectomy. Case Report

BACKGROUND AND OBJECTIVES: Homologous blood transfusion risks are well known and some patients may refuse blood transfusions on religious grounds. This report aimed at describing a case of total proctocolectomy in Jehovah's Witness patient with $4 \mathrm{~g} / \mathrm{dL}$ hemoglobin.

CASE REPORT: Male patient, 17 years old, with family history of adenomatous polyposis. The disease was manifested at eight years of age, characterized by bleeding. At 13 years of age he was submitted to total colectomy. At 17 years of age he was submitted to total proctocolectomy. Patient was prepared with erythropoietin, folic acid, infusion of iron and vitamin $B_{12}$. Red blood cell count revealed $\mathrm{He}=4,200,000 / \mathrm{mm}^{3}$, hemoglobin $=10.5 \mathrm{~g} / \mathrm{dL}$, hematocrit $=37 \%$ platelets $=273,000 / \mathrm{mm}^{3}$ and normal prothrombin time. Patient was continuously monitored with NIBP, pulse oximetry, capnography and ECG. Anesthesia was induced with propofol, sufentanil, pancuronium and enflurane in closed system. Patient received 7,000 $\mathrm{mL}$ lactated Ringer's and $150 \mathrm{~mL}$ of $20 \%$ human albumin. Total diuresis was $2,900 \mathrm{~mL}$. Surgery lasted 10 hours and 30 minutes. Patient was referred to the ICU with $20 \%$ hematocrit, 2,300,000/ $\mathrm{mm}^{3}$ red cells, 4,2 g/dL hemoglobin and was maintained with propofol and atracurium. Next day evaluation revealed $18 \%$ hematocrit, $2,050,000 / \mathrm{mm}^{3}$ red cells and $4 \mathrm{~g} / \mathrm{dL}$ hemoglobin. Patient was extubated 18 hours after surgery and was referred to the ward. Patient started eating four days after surgery and was discharged the $10^{\text {th }}$ postoperative day. Thirty days later patient presented $35 \%$ hematocrit, $4,000,000 / \mathrm{mm}^{3}$ red cells and 9.5 $\mathrm{g} / \mathrm{dL}$ hemoglobin. Six months later he returned for ileostomy closing. Patient was submitted to 12 surgeries without a single blood transfusion.

CONCLUSIONS: A good planning of the whole team (clinician, surgeon, anesthesiologist, intensive care staff) allows us to perform surgical procedures associated to major blood losses without administering blood.

Key Words: COMPLICATIONS: anemia; DISEASES: familial adenomatous polyposis; TRANSFUSION: Jehovah's witness

\section{INTRODUÇÃO}

$\mathrm{D}$ versas técnicas têm sido utilizadas para o tratamento da anemia em pacientes Testemunhas de Jeová. Em pacientes que recusam qualquer componente sangüíneo, o transporte de oxigênio, a liberação de oxigênio tecidual e a coagulação sangüínea podem ficar comprometidos durante grandes perdas de sangue. Um nível mínimo de hemoglobi- 
na no peri-operatório é um importante fator para reduzir as transfusões sangüíneas ${ }^{1,2}$. A questão fundamental é a definição do valor crítico da hemoglobina, que até hoje não foi estabelecido ${ }^{2,3}$. Será relatado um caso de grande perda sangüínea, resultando num nível de hemoglobina de $4 \mathrm{~g} / \mathrm{dL}$.

\section{RELATO DO CASO}

Paciente do sexo masculino, 17 anos, Testemunha de Jeová, $57 \mathrm{~kg}$ e $165 \mathrm{~cm}$. Início da doença aos oito anos, manifestada por hematoquezia com ressecção de três pólipos por retossigmoidoscopia, sendo diagnosticada polipose colônica. Dos oito aos 11 anos foram realizadas mais duas ressecções de pólipos intestinais por retossigmoidoscopia. Aos 12 anos, nova ressecção de pólipos por retossigmoidoscopia e laparotomia com colostomia. Aos 13 anos foi diagnosticado novo pólipo adenomatoso e o paciente submetido à colectomia total com anastomose íleo-retal. No mesmo ano evoluiu com presença de pólipos retais, que foram ressecados por via baixa. Aos 14 anos, nova ressecção por via baixa. Evoluiu com exteriorização de pólipos por via anal às evacuações, com pequeno sangramento, tendo sido submetido a dois procedimentos de ressecção aos 15 anos. Aos 16 anos, nova recidiva de pólipos retais com ressecção por via baixa. Aos 17 anos foi internado com quadro de hemorragia digestiva por polipose colônica, sendo indicada proctocolectomia com anastomose íleo-anal com bolsa ileal em jota, biópsia hepática e ileostomia. Preparado com eritropoietina (7.500U) três vezes por semana para estimular a eritropoiese, ácido fólico (5 mg) por dia, infusão de ferro $(200 \mathrm{mg})$ e vitamina $\mathrm{B}_{12}(1 \mathrm{~g})$ três vezes por semana até que o hematócrito atingisse $35 \%$ e/ou concentração de hemoglobina chegasse a $10 \mathrm{~g} / \mathrm{dL}$.

Os exames pré-operatórios indicavam contagem de hemácias de $4.200 .000 / \mathrm{mm}^{3}$, hemoglobina de $10,5 \mathrm{~g} / \mathrm{dL}$ e o hematócrito de $37 \%$. A contagem de plaquetas foi de $273.000 / \mathrm{mm}^{3}$, e o tempo de protrombina normal. A pressão arterial era de $105 \times 55 \mathrm{mmHg}$ e a freqüência cardíaca (FC) de 80 bpm.

A anestesia foi induzida com propofol, sufentanil e pancurônio e a manutenção com doses fracionadas de sufentanil, pancurônio e enflurano em circuito fechado. Um cateter $16 \mathrm{G}$ foi introduzido na veia do antebraço. Com o objetivo de manter a volemia e a pressão arterial, foi infundido um total de $7.000 \mathrm{~mL}$ de solução de Ringer com lactato e $150 \mathrm{~mL}$ de albumina humana a $20 \%$. O paciente foi continuamente monitorizado com relação à isquemia miocárdica na derivação CM5. A diurese durante o ato cirúrgico foi de $2.900 \mathrm{~mL}$. Não houve necessidade de infusão de qualquer substância vasopressora durante o ato operatório. Aduração da cirurgia foi de 10 horas e 30 minutos (Figura 1).

Após a cirurgia o paciente foi encaminhado à unidade de terapia intensiva (UTI) e admitido com PANI de $110 \times 70 \mathrm{mmHg}$, $\mathrm{FC}$ de 88 bpm, mantido em ventilação mecânica com $\mathrm{FiO}_{2}$ de 0,6 e PEEP de $5 \mathrm{~cm}$, sendo mantido com infusão contínua de propofol e atracúrio e monitorizado continuamente com cardioscoópio e oximetro de pulso. Nas primeiras 24 horas de internação não foram necessárias drogas vasopressoras adi-

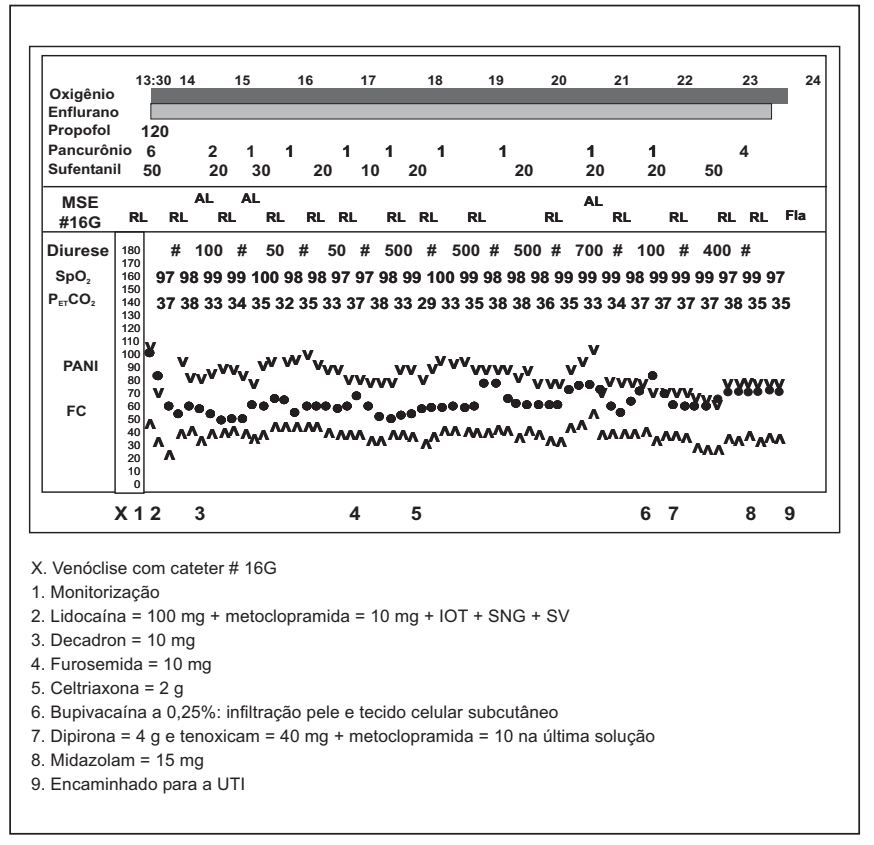

Figura 1 - Ficha de Anestesia

cionais. Na UTI foi acompanhado por um membro da equipe de anestesia e de cirurgia e, após coleta de amostra para determinação da série vermelha, foi proibida coleta de qualquer volume de sangue para exames complementares. O hematócrito de admissão na UTI foi de $20 \%$, a contagem de hemácias foi de $2.300 .000 / \mathrm{mm}^{3}$ e os níveis de hemoglobina de 4,2 $\mathrm{g} / \mathrm{dL}$. No segundo dia de pós-operatório foi feita nova coleta de sangue para exames hematológicos que revelaram hematócrito de $18 \%$, contagem de hemácias de $2.050 .000 / \mathrm{mm}^{3}$ e hemoglobina de $4 \mathrm{~g} / \mathrm{dL}$. Dezoito horas após o término da cirurgia, o paciente foi extubado com estabilidade hemodinâmica (PANI $=100 \times 80 \mathrm{mmHg}$ e $\mathrm{FC}=$ $110 \mathrm{bpm}$ ). A antibioticoterapia constou de metronidazol e ceftriaxona.

No segundo dia de pós-operatório o paciente foi transferido da UTI para o quarto. No quarto dia de pós-operatório iniciou dieta líquida. No $10^{\circ}$ teve alta para sua residência. Do segundo ao $10^{\circ}$ dias de pós-operatório não foi colhido qualquer volume de sangue para exame. Não houve nenhuma complicação no pós-operatório. No $30^{\circ}$ de pós-operatório os exames hematológicos foram hematócrito de $35 \%$, contagem de hemácias de 4.000.000/ $\mathrm{mm}^{3}$ e hemoglobina de $9,5 \mathrm{~g} / \mathrm{dL}$.

Seis meses após a cirurgia, o paciente foi submetido ao fechamento da ileostomia e realizada dilatação da anastomose íleo-anal. A equipe anestésico-cirúrgica foi a mesma que realizou as 12 cirurgias no paciente. Da primeira cirurgia aos oito anos até a última aos 18 anos o paciente nunca recebeu transfusão sangüínea.

\section{DISCUSSÃO}

Existem diversos relatos de sobrevida de pacientes cirúrgicos com valores baixos de hematócrito ${ }^{1,4,5}$, sendo que o me- 
nor valor de hemoglobina encontrado na literatura médica é de $1,1 \mathrm{~g} / \mathrm{dL}^{5}$. Diversos relatos, como o presente, têm sugerido que o paciente cirúrgico e crítico tolera níveis de hemoglobina e hemácias muito mais baixos do que se acreditava antigamente. Este caso é interessante por relatar várias alternativas terapêuticas.

A polipose intestinal familiar é uma doença hereditária caracterizada por mais de cem pólipos do tipo adenoma, que são elevações na mucosa semelhantes a uma verruga, localizados no intestino grosso (cólon e reto). É causada por defeito genético específico no gene APC (adenomatous polyposis coli), que está localizado no cromossomo 5 . Esses pólipos começam a aparecer, em geral, após a puberdade, entre $12 \mathrm{e}$ 18 anos, sendo que no presente caso, a primeira manifestação da doença ocorreu aos oito anos, tendo sido ressecados três pólipos por retossigmoidoscopia. A cirurgia ainda é o único tratamento efetivo para a polipose intestinal familiar, ou seja, a remoção de todo o intestino grosso, incluindo ou não o reto. Neste caso, aos 13 anos foi submetido à colectomia total e aos 17 anos, à proctocolectomia total com anastomose íleo-anal.

As Testemunhas de Jeová não admitem a administração de transfusão de sangue total, de concentrados de hemácias, concentrados de glóbulos brancos, de plasma e de plaquetas. Entretanto, o entendimento religioso não proíbe de modo absoluto o uso de componentes do sangue como a albumina, as imunoglobulinas, os preparados para hemofílicos, os preparados de fibrina, a autotransfusão (desde que não haja desconexão entre a retirada e a infusão do sangue), a eritropoietina e o transplante de órgãos. No presente relato a eritropoietina foi utilizada no pré-operatório e a albumina humana durante o ato cirúrgico.

Durante vários dias no pré-operatório o hematócrito permaneceu baixo por sangramento contínuo através dos pólipos intestinais e elevou-se lentamente após terapia com eritropoietina, ferro, vitamina $B_{12}$ e ácido fólico. Trinta dias foram suficientes para que o valor do hematócrito subisse acima do previamente determinado para a realização da cirurgia. O paciente foi encaminhado ao procedimento cirúrgico com hematócrito de $37 \%$ e concentração de hemoglobina de 10,5 $\mathrm{g} / \mathrm{dL}$.

Os riscos associados com a transfusão sangüínea, de natureza infecciosa ou imunológica, continuam sendo os maiores questionamentos durante seu uso. Em virtude de seus benefícios e malefícios, a transfusão sangüínea tem mudado bastante nas últimas décadas. Existe um consenso mundial em restringir a necessidade de sangue para o mínimo necessário ${ }^{6}$. Entretanto, qual é este mínimo necessário? Os valores de $10 \mathrm{~g} / \mathrm{dL}$ de hemoglobina e $30 \%$ de hematócrito não são mais apropriados e as pesquisas continuam ${ }^{7}$. Desta forma, o valor mínimo da concentração de hemoglobina que indica a necessidade de transfusão sangüínea na prática clínica diária tem estimulado os programas de conservação de sangue $^{8}$. O valor da concentração de hemoglobina de $7 \mathrm{~g} / \mathrm{dL}$ é aceito na maioria dos estudos que determinam a necessidade de transfusão sangüínea ${ }^{9}$. Estudos em diferentes animais demonstraram que o valor crítico da hemoglobina está em torno de $4 \mathrm{~g} / \mathrm{dL}$. Hemodiluição normovolêmica com hemoglobina de $5 \mathrm{~g} / \mathrm{dL}$ foi bem tolerada em voluntários ${ }^{10}$. Extrema hemodiluição com nível de hemoglobina de $4 \mathrm{~g} / \mathrm{dL}$ em Testemunha de Jeová resultou em óbito 12 horas após a cirurgia. No presente relato não ocorreu óbito com igual valor de hemoglobina $(4 \mathrm{~g} / \mathrm{dL})$ e isto pode perfeitamente ser explicado pelas diferenças entre os pacientes de 84 anos versus 17 anos, sangramento do estômago por tumor maligno versus sangramento leve por polipose intestinal com colectomia total anterior, concentração inicial de hemoglobina de 7,7 $\mathrm{g} / \mathrm{dL}$ versus $10,5 \mathrm{~g} / \mathrm{dL}$.

Medidas da concentração de hemoglobina são facilmente realizadas, amplamente aceitas e freqüentemente usadas, mas elas são apenas um de vários métodos possíveis de indicação de transfusão sangüínea ${ }^{11}$. Atualmente, a avaliação da oxigenação dos tecidos é um importante fator na determinação da transfusão. O nível crítico de hemodiluição em humanos, definido como o ponto no qual o consumo de oxigênio $\left(\mathrm{Vo}_{2}\right)$ começa a diminuir em razão da insuficiente liberação de oxigênio $\left(\mathrm{Do}_{2}\right)$, não é conhecido. A resistência vascular sistêmica, resistência vascular pulmonar, débito cardíaco (DC), $\mathrm{Do}_{2}, \mathrm{Vo}_{2}$ e $\mathrm{P}_{50}\left(\mathrm{Po}_{2}\right.$ no qual a hemoglobina é $50 \%$ saturada com oxigênio) podem ser calculados através de coletas de sangue arterial e venoso. O consumo de oxigênio pode ser calculado dividindo $\mathrm{Vo}_{2}$ por $\mathrm{Do}_{2}$. Como uma das condutas traçadas seria evitar qualquer coleta de sangue (arterial ou venoso) para exames, acreditou-se que a manutenção da liberação tissular de oxigênio durante a cirurgia (anemia normovolêmica) foi sempre normal, pois não houve alteração dos parâmetros hemodinâmicos (FC, PA) e ventilatórios (oximetria e capnografia). O mesmo aconteceu na UTI durante o tempo em que o paciente permaneceu com infusão contínua de propofol e atracúrio. A hemodiluição normovolêmica reduz a viscosidade sangüínea e, posteriormente, a resistência vascular sistêmica (RVS). A diminuição da RVS aumenta o DC sem aumentar o trabalho cardíaco ${ }^{12}$.

Voluntários humanos podem tolerar níveis de até $5 \mathrm{~g} / \mathrm{dL}^{9} \mathrm{em}$ razão dos mecanismos compensatórios sistêmicos e da microcirculação. Contudo, há pacientes em que fatores clínicos como a redução do débito cardíaco, diminuição da extração de oxigênio, alteração dos gases sangüíneos e aumento do consumo de oxigênio são importantes. Além disso, existe uma variabilidade individual. A taquicardia, a hipotensão postural e as alterações do segmento ST no eletrocardiograma são fatores que devem ser considerados. No caso relatado não houve alterações da FC nem do segmento ST do eletrocardiograma.

Várias complicações da transfusão sangüínea podem ser comuns após administração de outros produtos do sangue, como plasma fresco e plaquetas. Transfusão autóloga de sangue também não é isenta de riscos ${ }^{13}$. O número de pacientes moderadamente anêmicos que são submetidos à cirurgia é freqüentemente em torno de $20 \%{ }^{14}$, e os níveis baixos da concentração de hemoglobina no pré-operatório são os maiores fatores de indicação para a transfusão sangüínea

${ }^{15}$. No presente caso o paciente não estava anêmico e a concentração de hemoglobina no final da cirurgia foi de $4,2 \mathrm{~g} / \mathrm{dLe}$ 
$4 \mathrm{~g} / \mathrm{dL}$ doze horas após. Um valor muito baixo de hemoglobina pode ser tolerado sem causar isquemia dos órgãos e sem ocorrer depressão do segmento ST, fato que foi observado durante monitorização no intra e pós-operatório.

A decisão para transfundir um paciente não pode ser baseada apenas na concentração de hemoglobina ou no valor do hematócrito. O uso destes parâmetros inevitavelmente resulta em supertransfusão em alguns pacientes e subtransfusão em outros ${ }^{16}$. Erros laboratoriais, graus de hemodiluição e presença de perdas ocultas de sangue são diferentes fatores que tornam inadequado o valor da hemoglobina como parâmetro para iniciar uma transfusão. Diversos estudos compararam a eficácia de diferentes estratégias de transfusão baseada na concentração da hemoglobina. Muitos deles não demonstraram nenhuma redução da morbidade e da mortalidade no pós-operatório com estratégias para manutenção da concentração da hemoglobina com valores altos. Num estudo prospectivo ${ }^{17}$, comparando dois regimes de transfusão (restritivo - concentração de hemoglobina de 7 a $9 \mathrm{~g} / \mathrm{dL}$ versus liberal - concentração de hemoglobina de 10 a $12 \mathrm{~g} / \mathrm{dL}$ ) em 838 pacientes de UTI, a mortalidade no $30^{\circ}$ dia foi similar em ambos os grupos, mas a mortalidade no pós-operatório imediato foi mais alta no grupo liberal. Em estudo piloto comparando duas diferentes estratégias de transfusão em pacientes ortopédicos ${ }^{18}$, os autores demonstraram que para observar alguma diferença entre as duas estratégias seria necessário um total de 12.000 pacientes. Num estudo aleatoriamente encoberto com duas estratégias de transfusão (restritivo: concentração de hemoglobina de $7 \mathrm{~g} / \mathrm{dL}$ ou liberal: hemoglobina concentração de $10 \mathrm{~g} / \mathrm{dL}$ ), foi demonstrado que o regime restritivo foi tão seguro como o liberal nos pacientes politraumatizados internados em UTI ${ }^{19}$. Uma metanálise combinando os resultados de sete estudos aleatoriamente encobertos foi incapaz de detectar importância clínica na diminuição da mortalidade e infecção pós-operatória após transfusão sangüínea ${ }^{20}$. Apesar do nível baixo de hemoglobina de 4,2 g/dL no final na cirurgia e de $4 \mathrm{~g} / \mathrm{dL}$ no primeiro dia de pós-operatório não ocorreu nenhuma infecção durante a internação, assim como não foi observada deiscência da anastomose íleo-anal.

Em Testemunhas de Jeová submetidos à cirurgia que recusaram o uso de sangue ${ }^{21}$, a mortalidade aumentou significativamente em pacientes com doenças cardíacas após a diminuição dos níveis de hemoglobina de 10 a $11 \mathrm{~g} / \mathrm{dL}$ para 6 a 6,9 $\mathrm{g} / \mathrm{dL}$. Neste mesmo estudo, nos pacientes que não tinham doença cardíaca e que apresentaram níveis similares de hemoglobina, não houve aumento da mortalidade, o que está de acordo com outros autores ${ }^{17}$. No presente relato em paciente jovem (17 anos), o nível de $4 \mathrm{~g} / \mathrm{dL}$ de hemoglobina não ocorreu morbidade e/ou mortalidade neste tipo de cirurgia. A transfusão sangüínea é um tratamento médico. Exceto em casos de emergência, a decisão de transfundir componentes do sangue deve ser avaliada contrabalançando seus riscose benefícios. Uma importante observação deve ser lembrada, a limitada capacidade das células vermelhas estocadas, por diversos dias, em restaurar a capacidade de consumo de oxigênio $^{22}$. Este fenômeno está relacionado à diminuição da capacidade de 2,3 DPG, resultando em aumento da afinidade de hemoglobina pelo oxigênio. A transfusão autóloga é a técnica de transfusão que utiliza o próprio sangue do paciente. Entretanto, as Testemunhas de Jeová não aceitam a estocagem de seu próprio sangue para posterior infusão. Nesses pacientes o melhor é realizar a hemodiluição normovolêmica aguda (HNA), que consiste na retirada de um volume previamente determinado de sangue e reposição simultânea de solução cristalóide, colóide ou ambos ${ }^{12,23}$. Este sangue não pode ser separado do paciente e será reinfundido durante ou no final da cirurgia. Em crianças de sete a 17 anos submetidas à artrodese de coluna vertebral, a HNA reduziu a necessidade de sangue homólogo sem aumentar as complicações decorrentes da anemia ${ }^{24}$. No presente relato não foi realizada a HNA, e desta forma, não se pode afirmar que o nível de hemoglobina teria sido mais alto no final da cirurgia. O sistema de recuperação das células sangüíneas durante a cirurgia é uma técnica aceita pelas Testemunhas de Jeová. Um aparelho recebe o sangue coletado do paciente por meio de sucção durante a cirurgia misturando-o com solução fisiológica, separando as hemácias e removendo as impurezas. Estas células sangüíneas são então reinfundidas no paciente sem que haja perda de continuidade entre os apareIhos e o paciente. O aparelho não foi utilizado no paciente por não fazer parte do arsenal terapêutico do hospital.

\section{CONCLUSÕES}

1. O preparo pré-operatório é fundamental no manuseio de pacientes Testemunhas de Jeová.

2. As equipes de cirurgiões, de anestesiologistas e de terapia intensiva em comum acordo e com um mesmo propósito podem trabalhar com nível muito mais baixo de hemoglobina do que o classicamente recomendado.

3. A manutenção da volemia é de fundamental importância nestes pacientes.

4. O manuseio no pós-operatório deve seguir um protocolo rígido, com condutas anestésicas (sedação, relaxamento e suporte de oxigênio a $100 \%$ ).

5. Paciente jovem e sem nenhuma doença cardiocirculatória pode suportar nível mais baixo de hemoglobina.

6. A alta precoce da UTI (alta para o quarto no segundo dia de PO) é fundamental para evitar infecção.

7. Nível de hemoglobina de $4 \mathrm{~g} / \mathrm{dL}$ foi compatível com a cirurgia e a recuperação pós-operatória.

\section{AGRADECIMENTO}

ABruno Chaves Costa Moreira e sua família pela confiança depositada na equipe desde os oito anos de vida. 


\section{Extreme Intraoperative Hemodilution in Jehovah's Witness Patient Submitted Total Proctocolectomy. Case Report}

Luiz Eduardo Imbelloni, TSA, M.D.; Lúcia Beato, TSA, M.D.; Arídio Ornellas, M.D.; Carlos Roberto Junqueira Borges, M.D.

\section{INTRODUCTION}

Several techniques have been used to treat Jehovah's Witnesses anemia. In patients refusing any blood product, oxygen transportation, tissue oxygen release and blood coagulation may be impaired during major blood losses. A minimum level of preoperative hemoglobin is the key to decrease blood transfusions $^{1,2}$. A major question is the definition of critical hemoglobin value, which has not yet been established ${ }^{2,3}$. This report aimed at describing a case of major blood loss resulting in $4 \mathrm{~g} / \mathrm{dL}$ hemoglobin.

\section{CASE REPORT}

Male patient, 17 years old, Jehovah's Witness, $57 \mathrm{~kg}$ and 165 $\mathrm{cm}$. Disease has started at 8 years of age and was manifested by hematochezia with resection of three polyps by retosygmoidoscopy, being diagnosed as colonic polyposis. From 8 to 11 years of age, patient was submitted to two additional intestinal polyp resection by retosygmoidoscopy, At 12 years of age he was submitted to new polyp resection by retosygmoidoscopy and to laparotomy with colostomy. At 13 years of age a new adenomatous polyp was diagnosed and patient was submitted to total colectomy with ileo-rectal anastomosis. In this same year, patient evolved with rectal polyps which were resected by anal route. At 14 years of age patient was submitted to a new anal route resection.

Patient evolved with anal exteriorization of polyps during evacuations, with minor bleeding, and was submitted to two resections at 15 years of age. At 16 years of age there was new recurrence of rectal polyps with resection. At 17 years of age patient was admitted with digestive hemorrhage by coIonic polyposis being indicated proctocolectomy with ileo-anal anastomosis and ileal pouch in "J", liver biopsy and ileostomy. Patient was prepared with erythropoietin $(7,500$ U) three times a week to stimulate erythropoiesis, folic acid (5 $\mathrm{mg} /$ day), iron (200 $\mathrm{mg}$ ) and vitamin $\mathrm{B}_{12}(1 \mathrm{~g})$ infusion three times a week until hematocrit was $35 \%$ and/or hemoglobin concentration reached $10 \mathrm{~g} / \mathrm{dL}$.

Preoperative tests indicated $4.200 .000 / \mathrm{mm}^{3}$ red cells, 10.5 $\mathrm{g} / \mathrm{dL}$ hemoglobin, $37 \%$ hematocrit, $273.000 / \mathrm{mm}^{3}$ platelets and normal prothrombin time. Blood pressure was $105 \times 55$ $\mathrm{mmHg}$ and heart rate $(\mathrm{HR})$ was $80 \mathrm{bpm}$.

Anesthesia was induced with propofol, sufentanil and pancuronium, and was maintained with incremental doses of sufentanil, pancuronium plus enflurane in closed circuit. A $16 \mathrm{G}$ catheter was introduced in a forearm vein. Aiming at maintaining volume and blood pressure, a total of $7,000 \mathrm{~mL}$ lactated Ringer's and $150 \mathrm{~mL}$ of $20 \%$ human albumin were infused. Patient was continuously monitored for myocardial ischemia at CM5 lead. Perioperative diuresis was $2,900 \mathrm{~mL}$.

No vasopressors drugs were needed during surgery, which lasted 10 hours and 30 minutes (Figure 1).

Patient was referred to the ICU and was admitted with NIBP of $110 \times 70 \mathrm{mmHg}$, HR of $88 \mathrm{bpm}$ and was maintained under mechanical ventilation with $0.6 \mathrm{FiO}_{2}$ and $5 \mathrm{~cm}$ PEEP. Patient was maintained with continuous propofol and atracurium infusion and was continuously monitored with cardioscopy and pulse oximetry. No vasopressors were needed in the first 24 postoperative hours. Patient was followed up in the ICU by a member of the anesthetic-surgical team and, after sample collection to determine red series, any collection of blood for additional tests was prohibited.

Hematocrit at ICU admission was $20 \%$, red cells were $2,300,000 / \mathrm{mm}^{3}$ and hemoglobin levels were $4.2 \mathrm{~g} / \mathrm{dL}$. New blood sample was collected in the $2^{\text {nd }}$ postoperative day for hematological tests, which revealed $18 \%$ hematocrit, $2,050,000 / \mathrm{mm}^{3}$ red cells and $4 \mathrm{~g} / \mathrm{dL}$ hemoglobin. Patient was extubated hemodynamically stable 18 hours after surgery $(\mathrm{NIBP}=100 \times 80 \mathrm{mmHg}$ and $\mathrm{HR}=110 \mathrm{bpm})$. Antibiotic therapy was achieved with metranidazole and ceftriaxone.

Patient was referred to the ward in the $2^{\text {nd }}$ postoperative day. In the $4^{\text {th }}$ postoperative day patient started liquid diet and in the $10^{\text {th }}$ he was discharged home. No blood was collected from the $2^{\text {nd }}$ to the $10^{\text {th }}$ postoperative day for tests. There were no postoperative complications. In the $30^{\text {th }}$ postoperative day hematological results were $35 \%$ hematocrit, $4,000,000 / \mathrm{mm}^{3}$ red cells and $9.5 \mathrm{~g} / \mathrm{dL}$ hemoglobin.

Six months after surgery patient was submitted to ileostomy closing and ileo-anal anastomosis dilatation. The same anesthetic-surgical team performed all 12 surgeries undergone by the patient. From the first surgery at 8 years of age to the

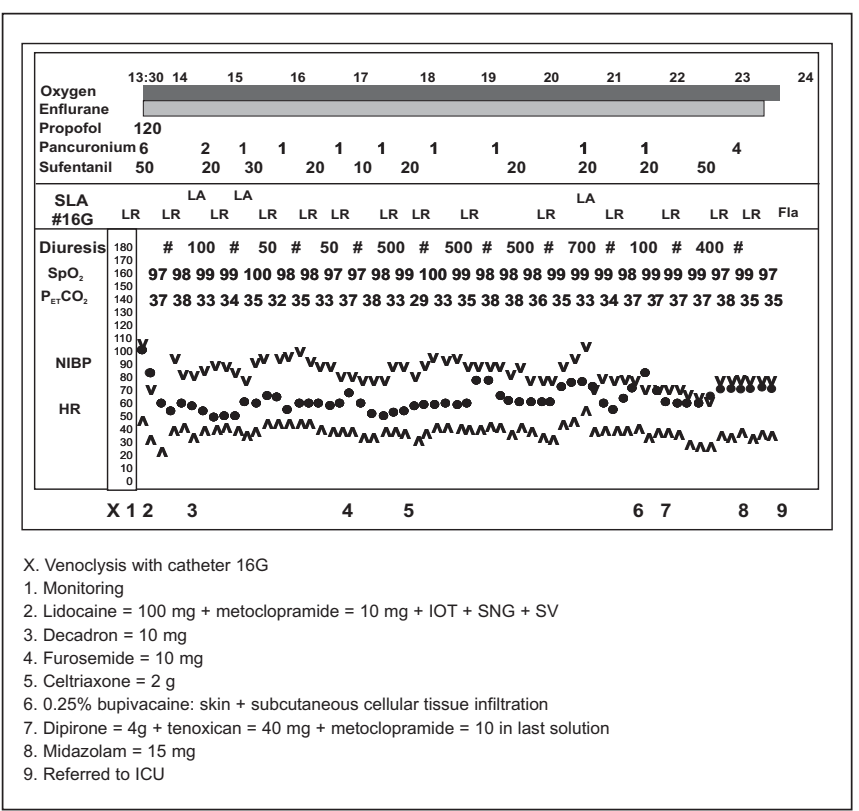

Figure 1 - Anesthetic Record 
last at 17 years of age, the patient has never received blood transfusion.

\section{DISCUSSION}

There are reports on survival of surgical patients with low hematocrit values ${ }^{1,4,5}$, and the lowest hemoglobin value in the literature is $1.1 \mathrm{~g} / \mathrm{dL}^{5}$. Many reports, as ours, have suggested that surgical and critical patients tolerate much lower hemoglobin and red cell levels than it has been previously believed. This is an interesting case because it reports different therapeutic alternatives.

Familial intestinal polyposis is a hereditary disease characterized by more than 100 adenoma-type polyps, which are mucosal elevations similar to warts located in the large intestine (colon and rectum). It is caused by specific genetic defect on APC gene (adenomatous polyposis coli), located in chromosome 5 . In general, these polyps appear after puberty, between 12 and 18 years of age, but in our case the first manifestation was at 8 years of age when 3 polyps were resected by retosygmoidoscopy. Surgery is still the sole effective treatment for familial intestinal polyposis, that is, removal of all large intestine, including or not the rectum. In our case, patient was submitted to total colectomy at 13 years of age and to total proctocolectomy with ileo-anal anastomosis at 17 years of age.

Jehovah's Witnesses do not admit total blood, red cells, leukocyte concentrate, plasma or platelets transfusion. Religious understanding, however, does not absolutely prohibit the use of blood products such as albumin, immunoglobulins, fibrin preparations, self-transfusion (provided there is no disconnection between blood removal and infusion), erythropoietin and organ transplantation. In our case, erythropoietin was used in the preoperative period and human albumin was used during surgery.

During several preoperative days hematocrit has remained low due to continuous bleeding through intestinal polyps and has slowly increased after therapy with erythropoietin, iron, vitamin $B_{12}$ and folic acid. Thirty days were enough for hematocrit value to increase above what was previously determined to perform the procedure. Patient was referred to surgical procedure with $37 \%$ hematocrit and $10.5 \mathrm{~g} / \mathrm{dL}$ hemoglobin concentration.

Infectious or immune risks associated to blood transfusion are still the major questions about its use. Due to benefits and disadvantages, blood transfusion has deeply changed in the last decades. There is a world consensus to limit the need for blood to a necessary minimum ${ }^{6}$. However, which is this necessary minimum? Values of $10 \mathrm{~g} / \mathrm{dl}$ hemoglobin and $30 \%$ hematocrit are not the best and researches continue ${ }^{7}$. So, minimum hemoglobin concentration indicating the need for blood transfusion in our daily practice is encouraging blood conservation programs ${ }^{8}$.

Value of $7 \mathrm{~g} / \mathrm{dL}$ hemoglobin concentration is accepted by most texts determining the need for blood transfusion ${ }^{9}$. Different animal studies have shown that critical hemoglobin level is approximately $4 \mathrm{~g} / \mathrm{dL}$. Normovolemic hemodilution with $5 \mathrm{~g} / \mathrm{dL}$ hemoglobin was well tolerated by volunteers ${ }^{10}$. Extreme hemodilution with $4 \mathrm{~g} / \mathrm{dL}$ hemoglobin in Jehovah's Witness has resulted in death 12 hours after surgery. In our case there has been no death with the same hemoglobin value $(4 \mathrm{~g} / \mathrm{dL})$ and this may be easily explained by differences between patients aged 84 and 17 years, stomach bleeding by malignant tumor and mild bleeding by intestinal polyposis with previous total colectomy, initial hemoglobin concentration of $7.7 \mathrm{~g} / \mathrm{dL}$ and $10.5 \mathrm{~g} / \mathrm{dL}$, respectively.

Hemoglobin concentration measures are easy, widely accepted and often used, but they are just one among different possible methods to indicate blood transfusion ${ }^{11}$. Currently, tissue oxygenation is a major factor to determine transfusion. Critical hemodilution level in humans, defined as the point in which oxygen consumption $\left(\mathrm{Vo}_{2}\right)$ starts to decrease as a function of insufficient oxygen release $\left(\mathrm{Do}_{2}\right)$, is still unknown. Systemic vascular resistance, pulmonary vascular resistance, cardiac output (CO), $\mathrm{Do}_{2}, \mathrm{Vo}_{2}$ e $\mathrm{P}_{50}\left(\mathrm{Po}_{2}\right.$ in which hemoglobin is $50 \%$ saturated with oxygen) may be calculated from arterial or venous blood.

Oxygen consumption may be calculated dividing $\mathrm{Vo}_{2}$ by $\mathrm{Do}_{2}$. Since one of the approaches was to avoid any blood collection (arterial or venous) for tests, we believed that the maintenance of tissue oxygen release during surgery (normovolemic anemia) was always normal since there were no changes in hemodynamic (HR and BP) and ventilatory (oximetry and capnography) parameters. The same was true in the ICU during the time patient remained with continuous propofol and atracurium infusion. Normovolemic hemodilution decreases blood viscosity and then systemic vascular resistance (SVR). Decreased SVR increases CO without increasing cardiac work ${ }^{12}$.

Human volunteers may tolerate up to $5 \mathrm{~g} / \mathrm{dL}^{9}$ due to compensatory systemic and microcirculation mechanisms. However clinical factors such as decreased cardiac output, decreased oxygen extraction, changes in blood gases and increased oxygen consumption are also important. In addition, there is individual variability. Tachycardia, postural hypotension and ST-segment changes at ECG are factors to be considered. In our case there were no HR or ST-segment changes.

Several blood transfusion complications may be common after the administration of other blood products, such as fresh plasma and platelets. Autologous blood transfusion is also not risk-free ${ }^{13}$. The number of moderately anemic people submitted to surgery is approximately $20 \%{ }^{14}$ and low preoperative hemoglobin concentrations are major factors to indicate blood transfusion ${ }^{15}$. In our case, patient was not anemic, hemoglobin concentration at surgery completion was 4.2 $\mathrm{g} / \mathrm{dL}$ and twelve hours later it was $4 \mathrm{~g} / \mathrm{dL}$. A very low hemoglobin level may be tolerated without organs ischemia and without ST-segment depression, fact which has been observed during intra and postoperative monitoring.

The decision to transfuse a patient cannot be solely based on hemoglobin concentration or hematocrit values. These parameters will invariably result in over transfusion for some patients and in under transfusion for others ${ }^{16}$. Laboratory mistakes, hemodilution levels and hidden blood losses are fac- 
tors invalidating hemoglobin as parameter to start transfusion.

Different studies have compared the efficacy of different transfusion strategies based on hemoglobin concentration. Many have shown no decrease in postoperative morbidity and mortality when high hemoglobin levels were maintained. In a prospective study ${ }^{17}$ comparing two transfusion regimens (restrictive - 7 to $9 \mathrm{~g} / \mathrm{dL}$ hemoglobin concentration versus liberal - 10 to $12 \mathrm{~g} / \mathrm{dL}$ hemoglobin concentration) in $838 \mathrm{ICU}$ patients, mortality at 30 days was similar for both groups, however immediate postoperative mortality was higher for the liberal group.

In a pilot study comparing two different transfusion strategies for orthopedic patients ${ }^{18}$, authors have shown that 12 thousand patients would be needed to show some difference between the strategies. A randomized double-blind study with two transfusion strategies (restrictive: $7 \mathrm{~g} / \mathrm{dL}$ hemoglobin concentration, or liberal: $10 \mathrm{~g} / \mathrm{dL}$ hemoglobin concentration) has shown that the restrictive regimen was as safe as the liberal regimen for multiple trauma patients admitted to the ICU ${ }^{19}$. A metanalysis combining the results of seven randomized double-blind studies was unable to detect clinical importance in the decrease of postoperative mortality and infection after blood transfusion ${ }^{20}$. Notwithstanding the low hemoglobin level of $4.2 \mathrm{~g} / \mathrm{dL}$ at surgery completion and of $4 \mathrm{~g} / \mathrm{dL}$ in the first postoperative day, there has been no infection or ileo-anal anastomosis dehiscence, during hospital stay.

In Jehovah's Witnesses submitted to surgeries and refusing blood ${ }^{21}$, mortality has significantly increased in cardiac patients after hemoglobin levels decrease from $10-11 \mathrm{~g} / \mathrm{dL}$ to 6 $6.9 \mathrm{~g} / \mathrm{dL}$. In this same study, mortality has not increased among patients with no heart disease and with similar hemoglobin levels, which is in line with other authors ${ }^{17}$. In our case of young patient ( 17 years old) and $4 \mathrm{~g} / \mathrm{dL}$ hemoglobin, there has been no morbidity and/or mortality.

Blood transfusion is a medical treatment. Except for emergency cases, the decision of transfusing blood products should be evaluated by balancing its risks and benefits. Amajor point to be reminded is the limited ability of red cells stored for several days to restore oxygen transportation capacity ${ }^{22}$. This phenomenon is related to decreased 2.3 DPG capacity resulting in increased affinity of hemoglobin for oxygen. Autologous transfusion is the technique using the blood of patients themselves.

Jehovah's Witnesses, however, do not accept the storage of their own blood for future use. The best for these patients is acute normovolemic hemodilution (ANH), which consists on removing a predetermined volume of blood and simultaneous replacing it with crystalloid, colloid or both ${ }^{12,23}$. This blood cannot be separated from the patient and will be reinfused during or after surgery. In children aged 7 to 17 years submitted to spinal arthrodesis, ANH has decreased the need for homologous blood without increasing anemia-related complications ${ }^{24}$. ANH was not used in our case, so it is impossible to state that hemoglobin levels would be higher at surgery completion.
Blood cells recovery process during surgery is a technique accepted by Jehovah's Witnesses. A device receives blood collected by suction from the patient during surgery and mixes it with saline solution separating red cells and removing impurities. These blood cells are then reinfused in the patient without loss of continuity between devices and patient. This device was not used in our patient for not being part of the institution's therapeutic armamentarium.

\section{CONCLUSIONS}

1. Preoperative preparation is critical to manage Jehovah's Witnesses patients.

2. Surgical, anesthetic and intensive care teams, in agreement and with the same objective, may work with much lower hemoglobin levels than those classically recommended.

3. Volume maintenance is paramount for these patients.

4. Postoperative management shall follow a strict protocol with anesthetic approaches (sedation, relaxation and $100 \%$ oxygen support).

5. Young patients with no cardiovascular disease may tolerate lower hemoglobin levels.

6. Early ICU discharge (discharge to the ward in the second $P O$ day) was fundamental to prevent infection.

7. Hemoglobin level of $4 \mathrm{~g} / \mathrm{dL}$ was compatible with surgery and postoperative recovery.

\section{ACKNOWLEDGMENT}

To Bruno Chaves Costa Moreira and his relatives for trusting our team since he was eight years old.

\section{REFERÊNCIAS - REFERENCES}

01. Spahn DR, Leone BJ, Reves JG et al - Cardiovascular and coronary physiology of acute isovolemic hemodilution: a review of monoxigen-carrying and oxygen-carrying solutions. Anesth Analg, 1994;78:1000-1021.

02. A report by the American Society of Anesthesiology Task Force on Blood Component Therapy: Practice for blood component therapy. Anesthesiology, 1996;84:732-747.

03. Leone BJ, Spahn DR - Anemia, hemodilution, and oxygen delivery. Anesth Analg, 1992;75:651-653.

04. Viele MK, Weiskopf RB - What can we learn about the need for transfusion from patients who refuse blood? The experience with Jehovah's Witnesses. Transfusion, 1994;34:396-401.

05. Zollinger A, Hager P, Singer T et al - Extreme hemodilution due to massive blood loss in tumor surgery. Anesthesiology, 1997;87:985-987.

06. Spahn DR, Casutt M - Eliminating blood transfusion: new aspects and perspectives. Anesthesiology, 2000;93:242-255.

07. Whitaker DK - Transfusion strategy. Eur J Anaesthesiol, 2001;18:493-494

08. Strang T, Whitaker DK - Blood conservation strategies in cardiac anaesthesia. Curr Opin Anaesthesiol, 1994;7:53-58. 
09. Van der Linden P - Transfusion strategy. Eur J Anaesthesiol, 2001;18:495-498.

10. Weiskopf RB, Viele MK, Feiner J et al - Human cardiovascular and metabolic response to acute, severe isovolemic anemia. JAMA, 1998;279:217-221.

11. Spahn DR - Perioperative transfusion triggers for red blood cells. Vox Sang, 2000;78:(Suppl2):163-166.

12. Hardy JF, Belisle S, Janvier $G$ et al - Reduction in requirements for allogeneic blood products: nonpharmacologic methods. Ann Thorac Surg, 1996;62:1935-1943.

13. Goldman M, Remy-Prince S, Trepanier A et al - Autologous donation error rates in Canada. Transfusion, 1997;37:523-527.

14. Goodnough LT, Brecher ME, Kanter MH et al - Transfusion medicine. Second of two parts - blood conservation. N Engl J Med, 1999;340:525-533.

15. Goodnough LT, Despotis GJ, Parvin CA - Erythropoietin therapy in patients undergoing cardiac operations. Ann Thorac Surg, 1997;64:1579-1580.

16. Goodnough LT, Brecher ME, Kanter MH et al - Transfusion medicine. First of two parts - blood transfusion. N Engl J Med, 1999;340:438-447.

17. Hebert PC, Wells G, Blajchman MA et al - A multicenter randomized controlled clinical trial of transfusion requirements in critical care. Transfusion Requirements in Critical Care Investigators, Canadian Critical Care Trials Group. N Engl J Med, 1999;340:409-417.

18. Carson JL, Terrin ML, Barton FB et al - A pilot randomized trial comparing symptomatic vs. hemoglobin-level-driven red blood cell transfusions following hip fracture. Transfusion, 1998;38:522-529.

19. McIntyre L, Hebert PC, Wells $G$ et al - Is a restrictive transfusion strategy safe for resuscitated and critically ill trauma patients? J Trauma, 2004;57:563-568.

20. McAlister FA, Clark HD, Wells PS et al - A Perioperative allogeneic blood transfusion does not case adverse sequelae in patients with cancer: a meta-analysis of unconfounded studies. Br J Surg, 1998;85:171-178.

21. Carson JL, Duff A, Poses RM et al - Effect of anaemia and cardiovascular disease on surgical mortality and morbidity. Lancet, 1996;348:1055-1060.
22. Fitzgerald RD, Martin CM, Dietz GE et al - Transfusing red blood cells stored in citrate phosphate dextrose adenine- 1 for 28 days fails to improve tissue oxygenation in rats. Crit Care Med, 1997;25-726-732.

23. Macuco MV, Carrenho JMX, Zambonato JF - Immediate effect of acute preoperative isovolemic hemodiluition on adult patients hematocrit. Rev Bras Anestesiol, 1998;48:475-487.

24. Oliveira GS, Tenorio SB, Cumino DO et al - Acute normovolemic hemodilution in children submitted to posterior spinal fusion. Rev Bras Anestesiol, 2004;54:84-90.

\section{RESUMEN}

Imbelloni LE, Beato L, Ornellas A, Borges CRJ - Manoseo de Grave Disminución de Hemoglobina en Paciente Joven, Testigo de Jehová, Sometido a la Proctocolectomia Total. Relato de Caso

JUSTIFICATIVA Y OBJETIVOS: Los riesgos de transfusión homóloga de sangre son bien conocidos y algunos pacientes recusan esta transfusión por motivos religiosos. El objetivo fue relatar un caso de proctocolectomia total en un paciente Testigo de Jehová donde el nivel de hemoglobina fue de $4 \mathrm{~g} / \mathrm{dL}$.

RELATO DEL CASO: Paciente masculino, 17 años, historia de poliposis intestinal familiar. Iniciada a los ocho años, caracterizada por sangramiento. A los 13 años colectomia total. A los 17 años proctocolectomia total. Preparado con eritropoietina, ácido fólico, infusión de hierro y vitamina $B_{12}$. El hemograma reveló: hematíes $4.200 .000 / \mathrm{mm}^{3}$, hemoglobina $10,5 \mathrm{~g} / \mathrm{dL}$ y hematócrito del $37 \%$. Plaquetas $273.000 / \mathrm{mm}^{3}$, tiempo de protrombina normal. Monitorización con PANI, oximetría de muñeca (pulso) capnografia y ECG continuamente. Anestesia con propofol, sufentanil, pancuronio y enflurano en circuito cerrado. Infusión de $7.000 \mathrm{~mL}$ de solución de Ringer con lactato y $150 \mathrm{~mL}$ de albúmina humana a $20 \%$. Diuresis de $2.900 \mathrm{~mL}$. Duración de 10 horas y 30 minutos. En la UTI Ht del $20 \%$, hematíes $2.300 .000 / \mathrm{mm}^{3}, \mathrm{Hb}$ de $4,2 \mathrm{~g} / \mathrm{dL}$ y mantenido con propofol y atracúrio. En examen al día siguiente reveló: Ht del 18\%, hematíes de $2.050 .000 / \mathrm{mm}^{3}, \mathrm{Hb}$ de $4 \mathrm{~g} / \mathrm{dL}$. Extubado 18 horas después del término de la cirugía. Al segundo día fue encaminado para el cuarto. Al cuarto día iniciada con alimentación por vía oral. Alta hospitalario al décimo día de PO. En el $30^{\circ} \mathrm{PO} \mathrm{Ht}$ del $35 \%$, hematíes de $4.000 .000 / \mathrm{mm}^{3}$ y $\mathrm{Hb}$ de $9,5 \mathrm{~g} / \mathrm{dL}$. Seis meses después, encerramiento de la ileostomia. Sometido a 12 cirugías sin transfusión sanguínea.

CONCLUSIONES: Una planificación de todo el equipo (clínico, cirujano, anestesista y médicos de terapia intensiva) permite realizar procedimientos quirúrgicos asociados con importantes pierdas sanguíneas, sin administración de sangre. 